\title{
HEALTH PROMOTION ACTIVITIES IN JAPAN
}

\author{
Tatsuya Takeshita and Kanehisa Morimoto
}

\begin{abstract}
In Japan, the spread of immunization and the development of antibiotics and antituberculosis drugs have dramatically decreased the mortality rate from infectious diseases since World War II. Alterations in health practices, such as decreasing the intake of salty foods and strict management of hypertension, have resulted in prominent decreases in the mortality rate from cerebrovascular diseases, especially from cerebral hemorrhage. However, we still have high death rates from malignant neoplasms and circulatory diseases, which may be partly prevented through improvement of health status. We discuss here about various health promotion activities recently reported in Japan, including prevention of smoking, promotion of exercise, improvement of nutrition, improvement of mental health status, and health risk appraisal. Finally we would like to stress the importance of comprehensive assessment of health status in order to encourage health promotion activities further. J Epidemiol, 1996 ; 6 : S165-S168.
\end{abstract}

health promotion, lifestyle, smoking, mental health

In Japan, the spread of immunization and the development of antibiotics and antituberculosis drugs have dramatically decreased the mortality rate from infectious diseases since World War II. We also had a very high mortality rate from cerebrovascular diseases just after the war. Since then, alterations in health practices, such as decreasing the intake of salty foods and strict management of hypertension, have resulted in prominent decreases in the mortality rate from cerebrovascular diseases, especially from cerebral hemornhage. The mortality rate from ischemic heart diseases has been low in Japan compared with other developed countries, probably because of a low intake of meat and saturated fat. However, a recent national survey on vascular diseases indicated elevations of serum cholesterol levels in all age groups of both sexes from 1980 to 1990 . During that period, systolic blood pressure decreased, especially in the older age groups, whereas diastolic blood pressure did not change, in all age groups. Therefore, we might experience increases in the incidence and mortality rates from ischemic heart diseases in the near future.

Although the age-adjusted mortality rate from malignant neoplasms has decreased in females, that in males has slightly increased in the last three decades. This sex-difference probably reflects differences in major health practices. Smoking rates among Japanese males were as high as $80 \%$ in 1965 and have gradually decreased to about $60 \%$ in recent years. In contrast, smoking rates among Japanese females have been as low as $15 \%$ for the last three decades. Likewise, the frequency of habitual alcohol drinking is as high as $40 \%$ among adult Japanese males but is very low among Japanese females.

Dietary habits and other lifestyles of the Japanese have been changing rapidly toward European styles. We have experienced great decreases in the age-adjusted mortality from gastric and cervical cancer. On the other hand, we will have further increases in mortality from lung, colon, pancreas, prostate and breast cancer in the next one or two decades. Health promotion activities to prevent these diseases are of great importance.

Under the Industrial Safety and Health Law, enacted in 1972, all companies with 50 or more employees have an obligation to have their employees take health checkups, including measurement of blood pressure, liver function, and serum lipids, at least once a year. The health checkups have contributed to the early detection of hypertension and to the prevention of apoplexy. The law for the Health and Medical Service for the Aged, enacted in 1982, also stresses the promotion of annual health checkups for residents in the community over the age of

Received and accepted December 5, 1995.

Department of Hygiene and Preventive Medicine, Osaka University School of Medicine, Osaka, Japan.

Address for correspondence : Kanehisa Morimoto, Department of Hygiene and Preventive Medicine, Osaka University School of Medicine, 2-2 Yamada-oka Suita Osaka, 565 Japan. 
40. The law has forced local government to increase the number of public health nurses since then.

Since 1979, the Ministry of Labor has proposed and promoted a "silver health plan," which intends to improve the health status of workers of middle and advanced age. In 1988, the law was revised for the purpose of maintaining and improving all workers' health. The revision of the law enabled healthcare staffs to take action to maintain and improve workers'comprehensive health status, both psychologically and physically, regardless of their age. This action is called the "total health plan" and has been carried out in numerous factories so far. In this paper, we would like to introduce many ways to improve the psychological and physical health of the Japanese.

\section{PREVENTION OF SMOKING}

As in other developed countries, cigarette smoking has been shown to be a major factor in the risk for lung cancer ${ }^{1)}$ and myocardial infarction ${ }^{2)}$ in Japan. The smoking rate among Japanese males has decreased from $80 \%$ in 1965 to slightly less than $60 \%$ at the present time. The smoking rate among Japanese females has been stable at around 15\%. Although most public spaces have prohibited smoking and have provided corners for smokers, many offices have not yet completely isolated such smoking comers. Because the smoking rates are especially high among younger people, health education and consultation directed toward youth are primary issues.

Among Japanese junior high school students, Ogawa et al. ${ }^{3)}$ reported smoking rates among males of $6.9 \%$ and among females of $1.2 \%$. Kawabata et al. ${ }^{4)}$ reported slightly higher rates. A recent nationwide survey ${ }^{5)}$ found further increases in males $(10.1 \%)$ and females $(3.5 \%)$ of the same age. Principal motives for such youngsters to start smoking involved smoking by their parents and/or siblings ${ }^{4)}$ and by friends $\mathrm{s}^{3-4)}$. Kawabata et al ${ }^{4)}$ suggested that school teachers and parents cooperate to prevent smoking among youth. They also proposed that prevention programs be introduced in elementary schools.

At the Osaka Cancer Prevention and Detection Center, Oshima and Nakamura ${ }^{6)}$ have conducted a smoking cessation contest annually since 1988 . Ozasa et al. ${ }^{7)}$ gave individual anti-smoking advice to male smokers visiting a health checkup clinic. Six months or one year later, cessation rates were significantly higher in the intervention group than in the control group when the participants' smoking index was less than 400 and when they had attempted to quit smoking in the past ${ }^{7-8}$. Ogawa et al. ${ }^{9)}$ reported that about $36 \%$ of the participants were still successful one year after the five-day plan to stop smoking. We need to spread this kind of activity because of the persistently high smoking rate among Japanese males.

\section{PROMOTION OF EXERCISE}

The important role of exercise in the improvement of health is stressed in the total health plan described above. Reductions in physical labor and the increasing use of automobile have contributed considerably to decreased physical activities among the Japanese. The decreased physical activities have probably contributed to some extent to the increases in noninsulin-dependent diabetes mellitus, hyperlipidemia, and obesity, especially "visceral fat accumulation"10).

Yanagibori et al. ${ }^{11)}$ reported that a 12-week exercise walking plan (45 min walking, 3 or 4 times per week) resulted in a significant increase in high-density-lipoprotein cholesterol (HDLC) and a significant decrease in the ratio of total cholesterol over HDLC. Tsuji et al. ${ }^{12)}$ provided instructions for a recommended increase in physical activities to obese female subjects. After the study period, the study group had a significant reduction in body mass index and waist-hip ratio compared with the levels before the study. The study group also had a significant decrease in blood sugar levels 30-120 min after glucose intake compared with the control group. Kishikawa et al. ${ }^{13)}$ also gave instructions for recommended physical activity levels to male employees and observed increases in physical strength and reductions in psychological symptoms such as insomnia and depression. Osaka Gas Company began health programs to promote physical activities 20 years ago. Those programs have resulted in an improvement in physical strength, an increase in habitual exercise, and a decrease in habitual smoking ${ }^{14}$.

Uchino et al. ${ }^{15)}$ provided either diet or exercise instructions to participants in a class for obese women in a public health center. A reduction in body weight and a decrease in body fat were found in both the diet and exercise groups. However, maintenance of the achieved weight reduction was difficult to accomplish, because $18-48 \%$ of the participants regained body weight six months to one year later.

\section{IMPROVEMENT OF NUTRITION}

Seki and Yamaguchi ${ }^{16)}$ reported an educational health program for persons with hypercholesterolemia. Instructions to restrict calorie and fat intake and to increase vegetable intake were provided. The study group achieved a decrease in serum cholesterol levels. Iso et al. ${ }^{17)}$ conducted a 1.5 -year community-based education program for persons with hypertension. The study participants were randomly allocated to an "intensive education group" or a "usual education group." Those in the intensive education group showed a $6 \mathrm{mmHg}$-greater decrease in mean systolic blood pressure than those in the usual education group at both six months and 1.5 years.

\section{IMPROVEMENT OF MENTAL HEALTH STATUS}

In Japan, many employees, such as department and section chiefs or engineers, work so hard at the cost of their health that some of them lose their lives from "Karoshi," which means sudden death due to overwork. According to Uehata ${ }^{18)}$, the 
main causes of "Karoshi" are apoplexy, acute heart failure, and myocardial infarction. About two thirds of the affected employees were working long hours. Maruyama et al. ${ }^{19)}$ found that department chiefs who worked 10 or more hours per day perceived 3 times as much stress as those who worked 9 or fewer hours per day.

Kawakami et al ${ }^{20)}$ investigated the effects of perceived job stress on symptoms of depression among male blue-collar workers in an electrical factory in Japan. Job unsuitability was significantly correlated with the occurrence of depression in the two years of the study. Based on a longitudinal survey of 11,121 workers, Sugisawa et al. ${ }^{21)}$ showed that both psychological work overload and late-night work had a significant effect on the development of mental disorders. Effects of mental stress on the increase of blood pressure ${ }^{22)}$ and on the occurrence of cardiovascular disease ${ }^{233}$ have been reported.

Most of the studies cited have focused on "ill" health effects of mental stress, such as symptoms of depression and anxiety. Maruyama et al. ${ }^{24)}$ developed a comprehensive working-life satisfaction scale. This scale was significantly correlated with health practices, occupational stress, physical health, and job position ${ }^{25}$. This "positive" health scale, combined with the traditional mental health scales that measure symptoms of depression and anxiety, would be useful in assessing workers' mental health and in promoting comprehensive health.

\section{HEALTH RISK APPRAISAL}

Health risk appraisal (HRA) tries to evaluate each individual's health risk by using previously reported epidemiological findings and the individual's lifestyle data and to recommend improvement of the lifestyle. In the United States, HRA was first developed in the 1960s, and the latest edition (Healthier People) was published in 1988. In Japan, Nakamura et al. ${ }^{26)}$ developed a Japanese version of HRA, based on Japanese epidemiological data and information on personal lifestyles. Takahashi et al ${ }^{27)}$ applied HRA to obese persons. The obesity index and serum lipid levels in the HRA group tended to be lower one year after the recommendations based on the HRA were given.

\section{COMPREHENSIVE DETERMINATION OF HEALTH STATUS}

A large body of epidemiological studies has proved that lifestyle and mental stress have significant effects on the development of major adult diseases such as cerebrovascular and cardiovascular diseases, cancer, and allergic diseases. It is important to develop biomarkers that accurately predict the risk for such diseases. Morimoto ${ }^{28)}$ showed that those with poor lifestyles had significantly higher levels of chromosome alterations in peripheral blood lymphocytes than did those with good lifestyles. Morimoto and Takeshita ${ }^{29)}$ demonstrated that habitual alcohol drinkers with aldehyde dehydrogenase
(ALDH2) deficiency had a significantly higher level of chromosome alterations than did habitual drinkers without the deficiency. Kusaka et al.$^{30)}$ found that those with poor lifestyles had significantly lower natural killer or lymphocyte-activated killer activities than did those with good lifestyles. This kind of study would be helpful in explaining to the public how poor lifestyles affect health status and the risk for diseases. These biomarkers would also help to confirm the effects of lifestyle changes.

In summary, it is of great importance in Japan to promote primary prevention such as a nutritionally balanced diet, obesity control, smoking cessation, moderate alcohol use, physical exercise, and control of mental stress. Japanese epidemiologists and other health professionals need to conduct further epidemiological studies related to lifestyle issues and to encourage many kinds of health promotion activities.

\section{REFERENCES}

1. Hirayama T. Cancer epidemiology in Japan. Environ Health Perspect $1979 ; 32: 11-15$.

2. Kodama K, Sasaki H, Shimizu M. Trend of coronary heart disease and its relationship to risk factors in a Japanese population : a 26-year follow-up, Hiroshima Nagasaki study. Jpn Circ J $1990 ; 54$ : 414-421.

3. Ogawa H, Tominaga S, Gellert G, Aoki K. Smoking among junior high school students in Nagoya, Japan. Int J Epid $1988 ; 17$ : 814-820.

4. Kawabata T, Maruya N, Hiyama T, et al. Smoking and alcohol drinking behavior among Japanese adolescents. Results from "Japan know your body study". Jpn J Publ Health $1991 ; 38: 885-899$ (in Japanese).

5. Ozaki Y, Minowa M. Nationwide survey of smoking prevalence among school students in Japan. Jpn J Publ Health $1993 ; 40: 39-47$ (in Japanese).

6. Oshima A, Nakamura M. Life-style and cancer prevention. Activities of the Department of Cancer Prevention, Osaka Cancer Prevention and Detection Center. Jpn J Cancer Clin 1990; $36: 268-274$ (in Japanese).

7. Ozasa K, Higashi A, Shiroeda O, Watanabe Y, Aoike A, Kawai K. A study of smoking cessation in a human dock setting. Jpn J Publ Health 1991 ; 38 : 45-51 (in Japanese).

8. Higashi A, Ozasa K, Watanabe Y, et al. Efficacy of smoking cessation instruction for general smokers at an annual physical examination. Jpn J Publ Health 1995 ; 42 : 313-321.

9. Ogawa H, Miyazaki K, Hayashi T. Effectiveness of the five-day plan to stop smoking. Jpn J Publ Health 1988 ; $35: 80-84$ (in Japanese).

10. Nakamura T, Tokunaga K, Shimomura I, et al. Contribution of visceral fat accumulation to the development of coronary artery disease in non-obese men. Atherosclerosis $1994 ; 107: 239-246$.

11. Yanagibori R, Kawakubo K, Gunji A. Effects of 12 wk- 
exercise walking on serum lipids, lipoproteins and apolipoproteins in middle aged women. Jpn J Publ Health $1993 ; 40: 459-467$ (in Japanese).

12. Tsuji M, Yamashita Y, Yamanaka C, Shigeta Y. Methods and significance of promoting physical activities in health center. J Publ Health Pract 1993 ; 57 : 287-291 (in Japanese).

13. Kishikawa K, Shono N, Ichiba M, Ienaga S, Kumagai S, Nishizumi M. Effects of increased physical activities on risk factors related to the development of adult diseases. Occup Health J $1995 ; 18: 56-63$ (in Japanese).

14. Ogino I. Health education in a city gas company. Occup Health J 1993 ; $16: 10-16$ (in Japanese).

15. Uchino $H$, Usami $T$, Honda $M$, et al. The effect of longterm group education for obese women in a public health center. Jpn J Publ Health 1991 ; 38 : 11 -19 (in Japanese).

16. Seki M, Yamaguchi T. Effectiveness of a nutritional education program in lowering blood cholesterol levels in a public health center. Jpn J Publ Health $1993 ; 40: 440$ 450 (in Japanese).

17. Iso $\mathrm{H}$, Shimamoto $\mathrm{T}$, Sankai $\mathrm{T}$, et al. A randomized controlled trial of intensive and usual community-based education for blood pressure control. Jpn J Publ Health 1994 ; 41 : 1015-1026 (in Japanese).

18. Uehata $T$. Long working hours and occupational stressrelated cardiovascular attacks among middle-aged workers in Japan. J Hum Ergol Tokyo 1991 ; 20 : 147-153.

19. Maruyama S, Kohno K, Morimoto K. A study of preventive medicine in relation to mental health among middlemanagement employees (part2) - Effects of long working hours on lifestyles, perceived stress and working-life satisfaction among white-collar middle-management employees —. Jpn J Hyg $1995 ; 50: 849-860$ (in Japanese).

20. Kawakami N, Haratani T, Araki S. Effects of perceived job stress on depressive symptoms in blue-collar workers of an electrical factory in Japan. Scand J Work Environ Health $1992 ; 18: 195-200$.
21. Sugisawa A, Uehata T, Sekiya E, et al. Mental health and its related factors among middle-aged male workers : an eighteen-month follow-up study. Sangyo-igaku 1994 ; 36 : 91-101 (in Japanese).

22. Kawakami N, Haratani T, Kaneko T, Araki S. Perceived job-stress and blood pressure increase among Japanese blue collar workers : one-year follow-up study. Ind Health $1989 ; 27: 71-81$.

23. Kayaba K, Yazawa Y, Natsume T, et al. The relevance of psychosocial factors in acute ischemic heart disease : a case-control study of a Japanese population. Jpn Circ J $1990 ; 54: 464-471$.

24. Maruyama S, Satoh H, Morimoto K. Relationship between working-life satisfaction, health practices and primary symptoms/problems. Jpn J Hyg 1991 ; 45 : 10821094 (in Japanese).

25. Maruyama S, Kohno K, Morimoto K. A study of preventive medicine in relation to mental health among middlemanagement employees (part 1). Relationship between lifestyles and working-life satisfaction. Jpn J Hyg 1994 ; $49: 887-901$ (in Japanese).

26. Nakamura M, Oshima A, Miura M. Estimation of the effects of smoking on the long term health risk-An application to health education-. Jpn J Cancer Clin $1990 ; 36$ : 319-325 (in Japanese).

27. Takahashi E, Yoshida K, T. K. Evaluation of health risk appraisal on obesity. Jpn J Ind Health 1994 ; 36 : 326-327 (in Japanese).

28. Morimoto $\mathrm{K}$. Chromosome alterations in peripheral lymphocytes as indices of lifestyle and genotoxicity. Int Arch Occup Environ Health 1993 ; 65 : S37-S41.

29. Morimoto K, Takeshita $\mathrm{T}$. Low $\mathrm{Km}$ aldehyde dehydrogenase (ALDH2) polymorphism, alcohol-drinking behavior, and chromosome alterations in peripheral lymphocytes. Env Health Perspect 1996 : (in press).

30. Kusaka Y, Kondou H, Morimoto K. Healthy lifestyles are associated with higher natural killer cell activity. Prev med $1992 ; 21: 602-615$. 